\title{
Novel Exact Solutions of the Extended Shallow Water Wave and the Fokas Equations
}

\author{
Serbay DURAN ${ }^{1, *}$, Berat KARAAGAC ${ }^{1}$ and Alaattin $E S E N^{2}$ \\ ${ }^{1}$ Adiyaman University, Faculty of Education, 020040 Adiyaman, Turkey \\ ${ }^{2}$ Inonu University, Department of Mathematics, 44280 Malatya, Turkey
}

\begin{abstract}
In this study, a Sine-Gordon expansion method for obtaining novel exact solutions of extended shallow water wave equation and Fokas equation is presented. All of the equations which are under consideration consist of three or four variable. In this method, first of all, partial differential equations are reduced to ordinary differential equations by the help of variable change called as travelling wave transformation, then Sine Gordon expansion method allows us to obtain new exact solutions defined as in terms of hyperbolic trig functions of considered equations. The newly obtained results showed that the method is successful and applicable and can be extended to a wide class of nonlinear partial differential equations.
\end{abstract}

\section{Introduction}

Over the run of humanity history; people are taken their attention on sciences or engineering because of their deeply curious nature. They believe that patterns in all of nature can be discovered with the help of using intellect. Where at the laws of the natural and physical world are generally modeled in the form of evolution equations, searching the solutions of evolution equations is become one of the main areas of scientific works [1]. During the years, various new techniques are developed for solving evolution equation [25].

The aim of this study, to investigate solution of extended Shallow Water Wave equation and Fokas equation with the help of the Sine Gordon expansion method which is a useful and popular tool for constructing exact solutions.

\section{Sine-Gordon expansion method}

This section is cover a simple definition of the Sine-Gordon expansion method. Firstly, let us take into consideration the general Sine-Gordon equation given as

$$
u_{x x}-u_{t t}=m^{2} \sin u
$$

\footnotetext{
* Corresponding author: sduran@adiyaman.edu.tr
} 
where $u=u(x, t)$ is the desired function and $m$ is a constant. The using of wave transformation $u(x, t)=U(\xi)(\xi=k x+l t)$ to the Eq. (2.1) leads to reduce Sine-Gordon equation to a nonlinear ordinary differential equation given in the form of

$$
U^{\prime \prime}=\frac{m^{2}}{k^{2}-l^{2}} \sin (U)
$$

where $k$ and $l$ refer the number of wave in a unit distance and the wave speed, respectively. When the both sides of Eq. (2.2) is multiplied by $U^{\prime}$ and integrated once, the following form is found

$$
\left(\frac{U}{2}\right)^{\prime}=\frac{m^{2}}{k^{2}-l^{2}} \sin ^{2}\left(\frac{U}{2}\right)+K
$$

where $K=0$. When $\frac{U}{2}=w(\xi)$ and $a^{2}=\frac{m^{2}}{k^{2}-l^{2}}$ are taken, the new form of the equation (2.3) is found as

$$
w^{\prime}=a \sin (w)
$$

When $a=1$ is taken, the Eq. (2.4), the basic form of Eq (2.1) is finally found as follows

$$
w^{\prime}=\sin (w) \text {. }
$$

Then, the solutions of the Eq. (2.5) can be found as follows

$$
\begin{aligned}
& \sin (w(\xi))=\left.\frac{2 p e^{\xi}}{p^{2} e^{2 \xi}+1}\right|_{p=1}=\sec h(\xi) \\
& \cos (w(\xi))=\left.\frac{p^{2} e^{2 \xi}-1}{p^{2} e^{2 \xi}+1}\right|_{p=1}=\tan h(\xi)
\end{aligned}
$$

Where the integration constant is taken as $p \neq 0$. Thus, the general form is expressed of nonlinear PDE with two variables as following

$$
P\left(u, u_{x}, u_{t}, u_{x x}, u_{x t}, u_{t t}, \ldots, x, t\right)=0
$$

and using the transformation given as

$$
u(x, t)=U(\xi), \quad \xi=k x+l t .
$$

we obtain a nonlinear ordinary differential equation (NODE) for $U(\xi)$

$$
G\left(U, U^{\prime}, U^{\prime \prime}, \ldots\right)=0 .
$$

In a similar way followed above, it is assumed that the solution $U(\xi)$ of the nonlinear equation given in Eq. (2.10) can be formed as follows

$$
U(\xi)=\sum_{i=1}^{m} \tanh ^{i-1}(\xi)\left[B_{i} \sec h(\xi)+A_{i} \tanh (\xi)\right]+A_{0} .
$$

Assuming that the wave solutions of Eq. (2.11) can be written in the following form:

$$
U(w)=\sum_{i=1}^{m} \cos ^{i-1}(w)\left[B_{i} \sin (w)+A_{i} \cos (w)\right]+A_{0}
$$


Where $A_{i}, B_{i},(0 \leq i \leq m)$ are desired coefficients and $m$ is positive integers. $m$ will be determine with homogeneous balance principle. After, using the Eq. (2.12) is substituted into Eq. (2.10), collecting all the terms according to powers of function $(\sec h(\xi) \tanh (\xi))^{i}$ or $(\sin (\xi) \cos (\xi))^{i}$ and at the last equalizing coefficients to zero, an algebraic equation system is obtained. When this new system is solved, the travelling wave solutions of Eq. (2.8) is obtained.

\section{Exact Solitary Wave Solutions of the Nonlinear Differential Equations}

\subsection{The (4+1) Dimensional Fokas equation}

Consider The (4+1) dimensional Fokas equation [6] given by:

$$
\begin{gathered}
4 u_{x t}-u_{x x y y}+u_{x y y y}+12 u_{x} u_{y}+12 u u_{x y}-6 u_{z w}=0 \\
u(x, y, z, w, t)=u(\phi), \quad \phi=\alpha x+\beta y+\gamma z+\lambda w+c t
\end{gathered}
$$

where $\alpha, \beta, \gamma$ and $\lambda$ are real parameters. Using the transformation given in Eq. (3.2) into Eq. (3.1), we get the following NODE;

$$
\left(\alpha \beta^{3}-\alpha^{3} \beta\right) \tilde{u}^{(4)}+(4 \alpha c-6 \gamma \lambda) \tilde{u}^{\prime \prime}+12 \alpha \beta\left(\tilde{u} \tilde{u}^{\prime}\right)^{\prime}=0 .
$$

And integrating twice with respect to $\xi$ Eq. (3.3) yields following equation,

$$
\left(\alpha \beta^{3}-\alpha^{3} \beta\right) \tilde{u}^{\prime \prime}+(4 \alpha c-6 \gamma \lambda) \tilde{u}+6 \alpha \beta \tilde{u}^{2}=0 .
$$

where $\tilde{u}^{2}$ is the highest order nonlinear term and $\tilde{u}^{\prime \prime}$ is the highest order derivative. Balancing them leads us to obtain $m=2$. Thus the solution is obtained as

$$
\tilde{u}(\phi)=A_{0}+A_{1} \tanh (\phi)+A_{2} \tanh ^{2}(\phi)+B_{1} \sec h(\phi)+B_{2} \tanh (\phi) \sec h(\phi)
$$

We insert Eq. (3.5) into Eq. (3.4), a polynomial of $\tanh (\phi), \operatorname{sech}(\phi)$, and also $\alpha, \beta, \gamma, \lambda$ is come out. Summing all the terms of the polynomial according to power of $\tanh (\phi), \operatorname{sech}(\phi)$, and equating whole summation to zero with the help of Wolfram Mathematica 9, gives us desired coefficients as

$$
A_{0}=\frac{1}{2}\left(-\alpha^{2}+\beta^{2}\right), A_{1}=B_{1}=0, A_{2}=\frac{1}{2}\left(\alpha^{2}-\beta^{2}\right), B_{2}=\frac{-i}{2}\left(\alpha^{2}-\beta^{2}\right), c=\frac{\alpha^{3} \beta-\alpha \beta^{3}+6 \gamma \lambda}{4 \alpha}
$$

substituting these coefficients into Eq. (3.5), produces the following solution:

$$
\begin{aligned}
& u(x, y, z, w, t)=\frac{1}{2}\left(-\alpha^{2}+\beta^{2}\right)+\tanh \left(\alpha x+\beta y+\gamma z+\lambda w+\frac{\left(\alpha^{3} \beta-\alpha \beta^{3}+6 \gamma \lambda\right) t}{4 \alpha}\right) \\
& \left(-\frac{i}{2}\left(\alpha^{2}-\beta^{2}\right) \sec h\left(\alpha x+\beta y+\gamma z+\lambda w+\frac{\left(\alpha^{3} \beta-\alpha \beta^{3}+6 \gamma \lambda\right) t}{4 \alpha}\right)\right. \\
& \left.+\frac{1}{2}\left(\alpha^{2}-\beta^{2}\right) \tanh \left(\alpha x+\beta y+\gamma z+\lambda w+\frac{\left(\alpha^{3} \beta-\alpha \beta^{3}+6 \gamma \lambda\right) t}{4 \alpha}\right)\right) .
\end{aligned}
$$



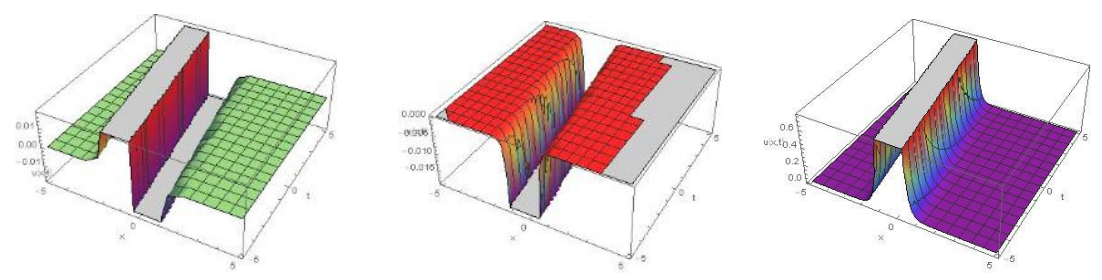

Fig. 1. The imaginary part, real part and Absolute solutions of Fokas equation by substituting the values $\alpha=5, \beta=0.1, \gamma=\mu=0.5-5<\mathrm{x}, \mathrm{t}<5$, respectively.

\subsection{Extended Shallow Water Wave Equation}

Extended shallow water wave equation reads [7]:

$$
\begin{gathered}
u_{x z t}+u_{x x y z}-2\left(u_{x x} u_{y z}+u_{y} u_{x x z}\right)-4\left(u_{x} u_{x y z}+u_{x z} u_{x y}\right)+\alpha u_{x y z}=0 \\
u(x, y, z, t)=\tilde{u}(\phi), \quad \phi=\beta x+\gamma y+\lambda z-c t
\end{gathered}
$$

where $\alpha, \beta, \gamma$ and $\lambda$ are real parameters, $c$ denotes wave velocity. Transformed form of Eq. (3.8) is

$$
\beta^{3} \gamma \lambda \tilde{u}^{\prime \prime \prime}+(\alpha \beta \gamma \lambda-\beta \gamma c) \tilde{u}^{\prime}-3 \beta^{2} \gamma \lambda\left(\tilde{u}^{\prime}\right)^{2}=0 .
$$

With the help of the balancing principle, we can found $m=1$. Thus, solution is obtained

$$
\tilde{u}(\phi)=A_{0}+A_{1} \tanh (\phi)+B_{1} \sec h(\phi)
$$

When the Sine- Gordon expansion method is applied, we get following coefficients and desired solutions given as

and

$$
A_{1}=-\frac{\sqrt{-\alpha \beta \lambda+c \lambda}}{\sqrt{\gamma \lambda}}, \quad B_{1}=\frac{\sqrt{\alpha \beta \lambda-c \lambda}}{\sqrt{\gamma \lambda}}, \quad \beta=\frac{\sqrt{-\alpha \beta \lambda+c \lambda}}{\sqrt{\gamma \lambda}}
$$

$$
\begin{aligned}
u(x, y, z, t)= & A_{0}+\frac{\sqrt{-\alpha \gamma \lambda+c \lambda}}{\sqrt{\gamma \lambda}} \sec h(\beta x+\gamma y+\lambda z-c t) \\
& -\frac{\sqrt{-\alpha \gamma \lambda+c \lambda}}{\sqrt{\gamma \lambda}} \tan h(\beta x+\gamma y+\lambda z-c t)
\end{aligned}
$$
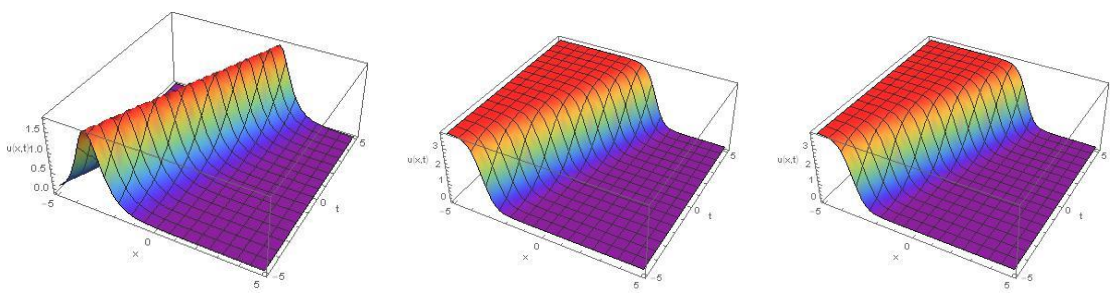

Fig. 2. The imaginary part, real part and Absolute solutions of shallow water wave equation by substituting the values $\alpha=0.5, \lambda=2, \gamma=0.25, \mu=0.5 \mathrm{c}=0.75, \mathrm{~A}_{0}=2,-5<\mathrm{x}, \mathrm{t}<5$, respectively. 


\section{Conclusions}

Under investigation in the present study, we investigate new solutions to the wellknown extended Shallow Water Wave equation and Fokas equation. Sine-Gordon expansion method is adopted to carry out this goal in a straightforward manner. With the assistance of the considered method, we construct novel and general solutions including hyperbolic trig functions. 3-dimensional of each solution obtained in the paper is illustrated in the study. As a result, the effectiveness and helpfulness of the Sine Gordon method deal with the nonlinear higher order equation is proved. The method can be used for constructing various nonlinear evolution equations in future studies.

\section{References}

1. A. Yokus and D. Kaya, Numerical and exact solutions for time fractional Burgers' equation, Journal Of Nonlinear Sciences And Applications 10.7, 3419-3428, (2017).

2. I.E. Inan, B. Kiliç, and S. Duran, $\mathrm{G}^{\prime} / \mathrm{G}$ expansion method and its applications to the 2 Dimensional Burgers equation and coupled Burgers type equation, World Appl. Sci. J. (2011).

3. E. M. E. Zayed and SA Hoda Ibrahim, Exact solutions of nonlinear evolution equations in mathematical physics using the modified simple equation method, Chinese Physics Letters 29.6 (2012).

4. A. Yokus, T. A. Sulaiman, H. Bulut, On the analytical and numerical solutions of the Benjamin-Bona-Mahony equation. Opt. Quant. Electron. 50, 31, (2018).

5. H. M. Baskonus and H. Bulut, On the complex structures of Kundu-Eckhaus equation via improved Bernoulli sub-equation function method, Waves in Random and Complex Media 25.4, 720-728 (2015).

6. A. M. Wazwaz, Multiple-soliton solutions for extended shallow water wave equations, Studies in Mathematical Sciences 1.1, 21-29 (2010).

7. H. Yinghui, Exact Solutions for-Dimensional Nonlinear Fokas Equation Using Extended F-Expansion Method and Its Variant, Mathematical Problems in Engineering 1-14, (2014). 